\title{
Necesidad de conceptos básicos para investigar en Matemática Financiera
}

\author{
Need to investigate basic concepts in Financial Mathematics \\ Luis Fernando Plaza Gálvez \\ Facultad de Ingeniería, Unidad central del Valle del cauca, Tuluá, Colombia \\ lplaza@uceva.edu.co
}

\begin{abstract}
Resumen- Por medio de esta propuesta, se presenta una reflexión sobre los procesos de enseñanza de Matemática Financiera, en los diversos programas de Ciencias Sociales, y la forma como los profesores que tienen la dirección del curso, brindan las herramientas necesarias para llevar a cabo una investigación en el área, luego de detectarse ausencia de conceptos básicos. La Matemática Financiera, debe ser mirada como un curso de origen en las ciencias exactas y no como un instrumento del formulismo, y dar la oportunidad a que sus instructores tengan formación académica de pregrado y/o posgrado en Matemática.
\end{abstract}

Palabras clave- Curriculum, Didáctica, Investigación, Matemática Financiera, Teoría APOE.

Abstract-By means of this proposal, it present a reflection on the teaching process of Mathematical Finance, in the various programs of Social Sciences, and the way the teachers with the direction of the course, provide the necessary tools to carry out an investigation in the area detected after absence of basic concepts. The Financial Mathematics, to be regarded as a source course in the exact sciences and not as an instrument of tokenism, and give the opportunity to have their instructors are academic undergraduate and $/$ or graduate in Mathematics.

Key Word - Curriculum, Didactics, Financial Mathematics, Research, Theory APOE.

\section{INTRODUCCIÓN}

Los estudiantes de programas académicos de Ciencias Sociales necesitan contar con fundamentos teóricos y conceptuales sobre Matemática Financiera que les permita mejorar sus procesos académicos. Por lo tanto analizar esas necesidades es el principal interés de esta investigación. Proponer una aproximación reflexiva, a partir del análisis histórico y epistemológico- cognitivo de los orígenes y conceptos de la Matemática Financiera a partir de los requerimientos de los estudiantes, para que su enseñanza permita una cultura investigativa en la comunidad estudiantil.

Para llevarlo a cabo es necesario identificar las necesidades básicas de los estudiantes que cursan Matemática Financiera desde el punto de vista de la historia de los conceptos, de un análisis epistemológico cognitivo y de las aplicaciones de la materia en la actualidad, así como ver la necesidad de implementar un curso que responda a las necesidades detectadas y determinar su viabilidad en términos institucionales y del proceso de aprendizaje de los alumnos. Es de anotar que lo anterior es debido en gran parte, al bajo contenido curricular del área de matemáticas en el componente básico en los programas de Ciencias Sociales.

\section{ORIGENES DEL PROBLEMA}

La falta de profundización en los orígenes de los conceptos básicos de la Matemática Financiera que se ofertan en los programas de Ciencias Sociales (Administración de Empresas, Contaduría Pública, Comercio Internacional, Finanzas, entre otros) en Colombia, hace que muchos de sus docentes no conozcan los orígenes de la disciplina, lo que puede generar debilidad al enseñar, al investigar y por supuesto al deducir fórmulas utilizadas en la matemática financiera. Situación que impide generar una cultura investigativa en el área por parte de sus estudiantes, convirtiéndola simplemente en una práctica de formulismos, o técnicas de solución supeditadas a una receta.

En el medio universitario colombiano, y tal vez en el iberoamericano, tomó fuerza, por razones de tipo académico, cultural y político-administrativo, el hecho que en las universidades sean asignadas las plazas docentes para los cursos de Matemática Financiera a profesionales con formación financiera y no a aquellos con formación matemática (como es el caso de los ingenieros), provocando una ausencia de producción académica e investigativa por parte de estudiantes y docentes en el área. Se ha identificado en los estudiantes que 
optan por estudiar programas como Administración de Empresas, Contaduría Pública, Finanzas y Comercio Internacional, que no tienen la mejor disposición a los contenidos matemáticos, y a su vez adolecen de argumentos, a partir de problemas relacionados con estas áreas, para dar sentido e iniciar una matematización que permita tratarlos de manera sistemática y general, eso los aleja de la posibilidad de desarrollar una modelación matemática de fenómenos financieros.

Recordando la baja formación en matemática mencionada antes, permite identificar la no presencia de varios conceptos básicos para asumir procesos en investigación del área. Los temas que se eluden son los que se relacionan con Series, Ecuaciones en Diferencias, Ecuaciones Diferenciales ordinarias y parciales, Ecuaciones diferenciales Estocásticas, así como con los Métodos Numéricos que permiten encontrar soluciones aproximadas en aquellos fenómenos que no se puedan describir, usando herramientas como las diferencias finitas y elementos finitos, en particular el estudio del mercado de Opciones Financieras [7].

Investigar en Matemática Financiera implica, el desarrollo de un currículo en el que se use la modelación y las herramientas matemáticas que se han ido incorporando en los últimos años, para llevar a cabo esta tarea de manera sistemática es necesario emprender un trabajo didáctico de investigación en el que se exploren cuidadosamente las necesidades de dicho currículo, por una parte, y por otra parte su diseño y prueba a través de la enseñanza en grupos de estudiantes de los programas de ciencias sociales antes mencionados.

\section{MOTIVACIÓN}

Es importante evidenciar la importancia (que no se le ha dado) de los procesos de enseñanza de la Matemática Financiera para lograr despertar un mayor interés por parte de sus actores y no limitarse al simple empleo de unas "recetas" o formulismos. Es necesario ir más allá y propiciar, tanto un ambiente de investigación en esta área, como también al interior del aula de clase.

Sería muy acertado que los estudiantes de los programas ya mencionados pudieran contar con una formación importante para optar a proyectos de investigación en Matemática Financiera, tal como hoy lo están haciendo docentes y estudiantes miembros de grupos de Investigación e Instituciones tales como el CIETyMA (Centro de Investigaciones en Economía Teórica y Matemática Aplicada) de la Universidad Nacional de San Martín (Argentina), el Departamento de Matemáticas de la Universidad de Buenos Aires, el CRM (Centre de Recerca Matemática) con sede en Barcelona (España), en el ITAM (México), el CMM (Centro de Modelamiento Matemático) de la Universidad de Chile (Santiago de Chile), ASEPUMA (Asociación Española de Profesores
Universitarios de Matemática para la Economía y la Empresa). Sin embargo, se considera que no basta reproducir en el contexto colombiano, las experiencias de otros países, si no que es importante potencializar los conocimientos que se tenían al respecto de las Matemáticas Financieras en las Ciencias Sociales teniendo en cuenta el conocimiento de la Educación Matemática con el que hoy se cuenta, que permita implementar el desarrollo de un currículo diseñado sobre la base de la modelación financiera en el salón de clases que haga posible que los estudiantes reflexionen sobre los conceptos matemáticos necesarios para resolver problemas de índole financiero y que los aprendan de manera significativa para que los puedan emplear en el ámbito laboral.

\section{APORTES DE LA INVESTIGACIÓN EN MATEMÁTICA FINANCIERA}

Al hacer un análisis de las personas que le han brindado a la economía un aporte desde las finanzas nos damos cuenta que muchas de ellas han tenido formación matemática. Este es el caso de los premios Nobel de Economía que se han obtenido entre los años 1969, y 2007. En las finanzas es necesario evidenciar los vínculos que se han encontrado con la física, tanto que se ha desarrollado una nueva área de investigación que se denomina Econofísica, la cual se originó en investigaciones que relacionan la Matemática Financiera, la Física y las Ecuaciones diferenciales [9]. Otro ejemplo de lo anterior se encuentra en el estudio de Matemática Financiera que ha permitido el desarrollo de tesis de Maestría con vínculos en Ecuaciones Diferenciales parciales como el caso del modelo de Black-Scholes [8].

En la investigación que se quiere desarrollar sobre el aprendizaje de Matemática Financiera, es importante evidenciar las necesidades de conocimiento fundamentalmente por parte de los alumnos, tanto desde el punto de vista cognitivo como desde el punto de vista del desarrollo de habilidades investigativas. Así mismo, el resultado del diagnóstico de necesidades hará posible una intervención curricular desde el punto de vista matemático y financiero que se propondrá a la luz de los resultados de la investigación.

\section{ESTADO DEL ARTE}

Se han encontrado, entre otros resultados, un análisis de naturaleza diagnóstico-descriptiva, en el que se ha concluido, entre otras cosas que los estudiantes de carreras de ciencias económicas de la Universidad Nacional de Villa María (Argentina), no estiman el análisis matemático como instrumento de apoyo académico, hasta tanto no se encuentre un vinculo con otra área de su campo de formación; ni como un fin, pues no se acogen al conocimiento matemático a la vez que no reconocen conceptos básicos del curso en mención [1]. 
Teniendo en cuenta la especial dificultad que supone a los alumnos, el aprendizaje de la Matemática Financiera, el equipo docente de esta disciplina en la facultad de Ciencias Económicas de la UNED (Universidad Nacional de Educación a Distancia) en España, decidió hace cinco años implantar una red de innovación docente, siendo los objetivos de esta iniciativa 1) facilitar el aprendizaje de los conceptos más complicados a través de la introducción de distintas técnicas metodológicas y, 2) adaptar paulatinamente las exigencias que propone la implantación de los grados en el nuevo espacio europeo de educación superior, y que al final permitió evidenciar, que los estudiantes registrados en dicho proyecto pudieron comprender con más facilidad los conceptos importantes de la materia en cuestión [6].

La búsqueda de otras investigaciones en Matemática Educativa relacionados con la enseñanza de las matemáticas financieras no ha sido fructífera, pues no ha permitido encontrar resultados relevantes. Esto pone en evidencia la necesidad de llevar a cabo sistemáticamente investigaciones en este campo que pongan énfasis no solamente en el diagnostico de necesidades, sino en el diseño curricular y su validación a través de la investigación seria, con base en las teorías y metodologías de la Educación Matemática.

\section{PREGUNTAS DE INVESTIGACIÓN}

Con base en esta primera búsqueda de antecedentes en la investigación, en las necesidades de replantear la forma en que las Matemáticas Financieras se enseñan en la Universidad, y del objetivo mencionado inicialmente, se pueden plantear las siguientes preguntas de investigación que permiten orientar el trabajo, las cuales podrán afinarse y reformularse con el avance de la investigación:

- ¿Cuáles son las necesidades de los alumnos que estudian programas académicos del área económica - administrativa en relación con las Matemáticas Financieras?

- ¿Qué requisitos debe cumplir un currículo diseñado con el fin de cubrir esas necesidades en una universidad específica en Colombia?

- Una vez intervenido un currículo, ¿Como los alumnos pueden aprender los conceptos esperados a través de la enseñanza de las Matemáticas Financieras con el currículo propuesto?

- Específicamente, ¿Cómo aprenden los estudiantes algunos de los conceptos más importantes relacionados con las Matemáticas Financieras?

\section{PROPUESTA DIDACTICA}

Cuando están de por medio las características culturales (como en el caso de la investigación-acción donde se tropieza con un objeto tecno-cultural) en el proceso de enseñanza de Matemática Financiera, se deben revisar las nociones expuestas en la Teoría Antropológica de lo Didáctico (TAD) [3]. El componente institucional (Noosfera) es importante a tener en cuenta cuando se pretende diseñar una propuesta didáctica. La Matemática Financiera se considera como una de las reformas a las matemáticas modernas, su enseñanza requiere de identificar una transposición didáctica que dé cuenta a los cambios que ha sufrido el saber enseñado y el saber sabio (de la matemática de las matemáticas) por parte de aquella que no lo son (financieras) [4].

El componente curricular, como origen del problema debe ser enfocado según lo expresa [12], teniendo claro que los objetivos se pueden apartar de los problemas éticos y políticos asociados al control de la educación, y se deben tener bien identificados los objetivos del estado, de quien desarrolla el Currículo, los del profesor y los del alumno.

La didáctica basada en el componente epistemológicocognitivo, juega un papel importante en el proceso de enseñanza de Matemática Financiera para estimular la formación investigativa y para eso se propone el empleo de la teoría APOE (Acción, Proceso, objeto y Esquema) [2, 10,13]. Incluso la didáctica va de la mano con el uso de una correcta bibliografía, que favorezca la adquisición de competencias esenciales propiciando la presencia de habilidades cognitivas, como componente articulado al Currículo expuesto antes.

A través de un diálogo entre estas dos teorías se ha puesto de manifiesto que son teorías más próximas de lo que en un primer acercamiento superficial podría parecer. Aún más, se ha mostrado cómo este diálogo ha dado frutos en términos de conceptos y metodologías que una teoría aporta a la otra sin contradecir ninguna de sus hipótesis fundamentales [14].

\section{METODO DE ANÁLISIS.}

La metodología a seguir será la propuesta en los marcos teóricos elegidos y será en su mayor parte de corte mixto, ósea cualitativo y cuantitativo, aunque es posible que en el análisis de necesidades se tome mano de algún método cuantitativo si se considera pertinente en el transcurso de la investigación. Se llevará a cabo un análisis histórico de los conceptos de la Matemática Financiera con el fin de tener clara su génesis y su papel en las aplicaciones de la disciplina.

A través de revisión de textos, de materiales de los alumnos que han cursado o cursan la asignatura Matemáticas Financieras en las universidades de la región (Valle del Cauca) se analizarán las praxeologías que se ponen en juego en su enseñanza y la 
transposición didáctica que de ellas se desprende. Mediante entrevistas a profesores que imparten la asignatura y a directivos de programas Matemáticas, Economía y Administración se complementará el análisis anterior para detectar las necesidades prácticas de la aplicación de las Matemáticas Financieras en la actualidad. Paralelamente, se diseñará una descomposición genética preliminar y se harán entrevistas a estudiantes que han cursado Matemáticas Financieras con el programa actual para determinar las construcciones que subyacen al aprendizaje de esta materia. Con base en los resultados de las entrevistas se refinará la descomposición genética particular para contar con un modelo que responda a las construcciones reveladas por el análisis histórico, la experiencia de los profesores y los resultados de las entrevistas a los alumnos.

Mediante los resultados del análisis realizado a través de la metodología de la TAD, y la descomposición genética, se diseñará una propuesta curricular y didáctica. Está se pondrá a prueba con el fin de validar que efectivamente se favorezca un mejor aprendizaje de los conceptos de la Matemática Financiera y el desarrollo de habilidades investigativas. Esta validación se llevará a cabo mediante un nuevo análisis del curso utilizando la metodología antes mencionada tanto en su parte institucional como en su parte cognitiva. Se compararán los resultados obtenidos por los estudiantes con quienes se haya puesto a prueba esta propuesta y aquellos entrevistados con anterioridad o se contrastarán los resultados con estudiantes de un grupo que no haya seguido el curso con la propuesta curricular diseñada.

Las investigaciones que se realizan en el campo de la educación matemática tienen dos objetivos: uno teórico, una mayor comprensión del pensamiento matemático y de los problemas que generan la enseñanza y aprendizaje de las mismas; y otro práctico, usar la comprensión anterior para mejorar la instrucción en matemáticas.

Dada la naturaleza de las Matemáticas Financieras se considera necesario que dichos cursos tengan un componente estimulante, en el cual se evidencie la posibilidad de su aplicación a situaciones reales, y se pueda observar las bondades de manejar el proceso de enseñanza - aprendizaje [11]. Esta última componente está ligada cercanamente al desarrollo tanto de la construcción significativa de los conceptos como del de las habilidades investigativas y de la componente institucional, pero es importante destacarla porque la consideramos una parte esencial a tomar en consideración en el diseño curricular.

\section{CONCLUSIONES}

Es importante notar las falencias con la que cuentan la mayoría de estudiantes de los curso de Matemáticas Financieras que hacen parte de los programas académicos en Ciencias Sociales, para enfrentar proyectos de investigación en el área, pues se demuestra que como parte del proceso cultural que imponen los directivos académicos en la mayoría de las veces, no permiten que dichos cursos sean guiados por matemáticos en formación, y contribuyan a generar y/o despertar nuevos conocimientos frutos de investigación. El análisis didáctico mediante la teoría APOE, ayuda a evidenciar este tipo de problemática.

\section{RECOMENDACIONES}

Se recomienda, iniciar una investigación de tipo cualitativo, con componente epistemológico - cognitivo, en la que se evidencie formalmente, las falencias que tienen los estudiantes para iniciar procesos investigativos, y a su vez, intentar mediante estructuras curriculares lograr despertar un mayor interés en los estudiantes por dichos procesos. Es importante considerar desde el punto de vista académico -administrativo, el permitir orientar los cursos de Matemática Financiera, a docentes con una buena formación en Matemáticas. En nuestro medio educativo colombiano, es importante considerar los tópicos expuestos en el Espacio Europeo de Educación Superior (EEES) [5].

\section{REFERENCIAS}

[1] R Abrate, I. Gabeitia, M. Pochulu. La enseñanza de la Matemática en Ciencias Económicas ¿en contexto o fuera de contexto?, UNIÓN. Revista Iberoamericana de Educación Matemática. 12. 53-62, (2007).

[2] J. Campero, M. Trigueros, Propuesta didáctica en optimización dinámica. Investigación en el aula. Educación Matemática, Santillana Distrito Federal, México. 22 (3). 87-117, (2010).

[3] Y. Chevallard, La transposición didáctica del saber al saber enseñado. AIQUE Grupo Editorial, 2a EDICIÓN, Buenos Aires, (1998).

[4] Y. Chevallard, El análisis de las prácticas docentes en la teoría antropológica de lo didáctico. Recherches en Didactique des Mathématiques. 19 (2). 221-266. Traducido por Ricardo Barroso Campos (Universidad de Sevilla) y Teresa Fernández Gracia (IES Martínez Montañés). (1999).

[5] M. F. García, E. J. Luque, B. Rodríguez, La enseñanza de las Matemáticas Financieras, Revista digital eXtoikos No. 4, (2011). Disponible en: http://www.extoikos.es/numero4.htm

[6] M. Hernández, D. de la Fuente, I. Pra, Innovación Docente en Matemática Financiera. Departamento de Economía y Contabilidad, Facultad de Ciencias Económicas y Empresariales, Universidad Nacional a Distancia UNED (España). Documento de trabajo, (2010).

[7] J. C. Hull, Options, Futures and other Derivatives. Ed. Prentice Hall. 6th edition. New Jersey, (2006).

[8] L. Plaza, Estudio Analítico - Numérico de la ecuación diferencial Parcial que origina el modelo de BLACKSCHOLES. Tesis de Maestría en Enseñanza de la Matemática. Universidad Tecnológica de Pereira, (2009).

[9] C. J. Quimbay, L. P. Salazar, Valoración de una Opción Europea con Volatilidad Estocástica usando la Integral de 
Camino de Feynman. Revista Colombiana de Física. 41 (2). $487-490$, (2009).

[10]H. Salgado, y M. Trigueros, "Conteo: una propuesta didáctica y su análisis”, Educación Matemática, 12 (1), 91-117, (2009).

[11] A. Sarmiento, J. M. Sánchez, Algunas cuestiones sobre la Enseñanza y el Aprendizaje de las Matemáticas en los estudios de Económicas y Empresariales. Departamento de Economía Aplicada II. Universidad de Coruña, (2007).

[12]L. Stenhouse L., La investigación como base de la enseñanza, Madrid, Ediciones Morata, (1987).

[13]M. Trigueros, La noción de Esquema en la investigación en Matemática Educativa a nivel superior. Educación Matemática, Santillana Distrito Federal, México 17 (1), 5-31, (2005).

[14] M. Trigueros, M. Bosch, y J. Gascón, (Por aparecer,) Actas del III Congreso Internacional sobre la Teoría Antropológica de lo didáctico. Sant Hilari Sacalm, España, 2011. 